19 Revue d'histoire du XIXe siècle

Société d'histoire de la révolution de 1848 et des

révolutions du XIXe siècle

47 | 2013

Quel est l'avenir du XIX ${ }^{\mathrm{e}}$ siècle ?

\title{
Le siècle de Baudelaire
}

\section{Yves Bonnefoy}

URL : https://journals.openedition.org/rh19/4539

DOl : $10.4000 /$ rh 19.4539

ISSN : $1777-5329$

\section{Éditeur}

La Société de 1848

\section{Édition imprimée}

Date de publication : 31 décembre 2013

Pagination : 29-35

ISSN : 1265-1354

Référence électronique

Yves Bonnefoy, "Le siècle de Baudelaire ", Revue d'histoire du XIXe siècle [En ligne], 47 | 2013, mis en ligne le 31 décembre 2016, consulté le 21 septembre 2021. URL : http://journals.openedition.org/ rh19/4539; DOI : https://doi.org/10.4000/rh19.4539 


\section{YVES BONNEFOY}

\section{Le siècle de Baudelaire}

Le XIX ${ }^{\mathrm{e}}$ siècle a vu se produire un des grands événements de l'histoire de l'esprit et nous a légué la tâche, guère assumée depuis - en tout cas nullement au plan où il faudrait que ce soit - d'en prendre mesure, d'en apprécier les dangers, d'en percevoir les apports possibles.

Cet événement est la banalisation de l'incroyance et l'effet que celle-ci a eu sur le travail des poètes. Lefranc de Pompignan ou Voltaire n'avaient guère de religion, au siècle précédent, mais ils n'avaient pas non plus beaucoup de poésie. Dans leurs poèmes peu avertis du jour après jour de leur existence le déclin du souci de Dieu allait du même pas que leur emploi de plus en plus résolument rhétorique de la parole rythmée dont ils tentaient de préserver l'apparence; et aux marges de cette littérature si satisfaite de soi la sensibilité proprement poétique, celle qui sait percevoir de la transcendance dans les situations et les choses de la réalité empirique, celle qui pourtant ne se sentait plus en mesure de prendre appui sur des articles de foi, avait alors à errer - étonnée, effrayée, peu capable pourtant encore d'un vrai ressaisissement dans les situations surprenantes de ces romans du temps que l'on dénommait gothiques.

Après quoi, aux approches du Romantisme et tout le temps qu'il dura, une sensibilité enfin lasse de l'excessive verbalité du classicisme tardif se voulut à nouveau vraie poésie, mais ce fut pour se laisser enflammer par une ferveur religieuse, ce qui retarda chez la plupart de ceux qui se voulaient des poètes la prise de conscience des changements qui se produisaient plus en profondeur dans l'esprit. Hugo, à la fin de sa vie, écrivait «La fin de Satan" et "Dieu", poèmes encore empreints du sentiment d'une surnature. Et le romantisme allemand avait éprouvé pour le lieu simplement terrestre, forêts, montagnes, un attrait dont le fond lui semblait être un divin qu'il identifiait au moyen de la croyance traditionnelle.

Tout autre chose ce qui se produit à partir des années 1840 chez des écrivains et des artistes dont les intuitions et le sentiment de responsabilité spirituelle n'étaient pourtant pas sans trouver du sens aux problèmes de la foi : mais c'était alors pour constater, cette fois consciemment et avec aussi bien 
quelque inquiétude, la syncope de celle-ci. Le plus intense et profond parmi les grands esprits de cette nouvelle époque, Baudelaire, se pose la question de l'existence de Dieu mais doit se résigner à comprendre qu'il ne croit pas, au moins en des moments qui sont au cœur de son attention. Il en ira de même, de façon plus tranchée mais pas pour autant plus radicale, chez Mallarmé, chez Rimbaud. Ces poètes savent garder leurs yeux sur les choses du proche, objets de vie quotidienne ou aspects de l'être sensible dans la profondeur desquels la perception d'une transcendance est un fait d'évidence simple, néanmoins la croyance en quoi que ce soit de plus que cette réalité qui se donne dans l'immédiat s'éteint en eux; et ce sont là, dans rien pourtant que quelques poèmes, des événements dont il y a lieu de penser qu'ils vont affecter très en profondeur la société tout entière.

Pourquoi? D'abord parce que cet effondrement du "principal pilier", comme dira Mallarmé, suggérait de comprendre que n'est que mythe, "glorieux mensonge ", ce qui était article de foi, et cela, c'est risquer, dangereusement, de perdre de vue la sorte de vérité qui se découvre dans la méditation des symboles. Faute que désormais l'on pressente et donc veuille et sache rejoindre le niveau le plus difficile de leur capacité signifiante, ceux-ci ne seront plus là pour permettre à quelques concepts de s'attacher à des aspects de la vie plus intimes que d'autres à son vœu assurément indicible, on ne saura plus retenir de l'instrument symbolique que son emploi par une imagination banalement désirante : si bien que beaucoup d'analogies, naguère foyers de symboles, se prêteront, par exemple, aux fantasmes les plus courtement sexuels, comme déjà dans la littérature "gothique». Un premier niveau embourbé de l'inconscient affleure dans la conscience : c'est le début d'une révision peut-être un peu trop précipitée des jugements de valeurs.

Et simultanément ou plutôt par voie de conséquence les sciences de la matière vont s'imaginer le champ libre, avec des effets secondaires proliférant sans contrôle dans une société invitée à se faire production et consommation d'objets eux-mêmes rien que matière, sans enracinement dans l'intériorité de la vie comme c'était encore le cas avec la table faite d'un bois dans les nœuds et fibres duquel la forêt respirait encore. Les idéologies vont pouvoir opposer leur goût de la généralité déserte, leur abstraction, à l'universel concret auquel on accède si la réflexion n'oublie pas la réserve de sens de l'instant vécu. Quand le siècle s'achève, avec ces trains qui sifflent dans les tunnels et même, bientôt, ces premiers avions qui décollent, c'est déjà la guerre moderne; et le totalitarisme prend pied dans la pensée politique. Les bouleversements qui affectent la relation à la transcendance auront des aspects qu'on peut estimer profondément bénéfiques mais aussi nombre de retombées qui d'évidence ne le sont pas.

Si bien qu'au moins en quelques esprits une certaine question bien précise peut prendre forme, qui confere à la poésie une fonction et une importance toutes nouvelles. Faut-il penser à la transcendance seulement en termes de 
surnature? N'est-il pas évident qu'une transcendance est tangible, et active, dans l'infini intérieur de rien qu'un brin d'herbe ou dans les suggestions que font à l'exister quotidien une simple écuelle sur une table ou des enfants à leur jeu, riant sur un chemin où peu à peu la nuit tombe? Et la transformation du rapport de l'humain à la transcendance devrait-elle fatalement se produire de la façon que l'on constata bientôt, un côtoiement sans approfondissement réciproque de la croyance traditionnelle, requise par des figures, des dogmes, des préceptes, et de cette incroyance qui, sensibilité autant que pensée, est tout de même fort susceptible de se laisser envahir par les mystères du monde et de la vie?

Cette question s'est posée, elle se pose évidemment aujourd'hui encore, et toujours plus. Et c'est dans son espace resté ouvert à bien des possibles que je prête attention aux contradictions dans lesquelles se débattit Baudelaire et suis prêt à penser que ce débat qui eut lieu en lui entre soi et soi - entre, dirai-je, le moi bâti par la pensée conceptuelle et le Je qui se souvient de l'unité qu'elle occulte et même censure - fut un creuset où brilla un or qu'il importe, et immensément, de percevoir. Cette attention à une œuvre de poésie m'incitant à penser que le XIX'e siècle a été, du point de vue que j'évoque et que je tiens pour fondamental, non pas seulement le siècle de Michelet ou, sur le tard, de Marx ou de Nietzsche et déjà presque de Freud, mais, en France en tout cas, celui de l'auteur des Fleurs du Mal.

Baudelaire, pourquoi? Eh bien, parce que si "Dieu est mort», comme on dit pour signifier le retrait du divin des significations et figures au moyen desquelles nous structurons la réalité empirique, il ne faut pas pour autant, j'y reviens, que se perde en cette dernière ou seulement s'y dilue le sentiment de la transcendance. Et la poésie est ce qui permet, ce qui seul permet, de répondre avec efficace à ce besoin de préservation. Dans un être au monde institué par des langues comme les nôtres occidentales, d'entrée de jeu hautement conceptualisées, les définitions de choses, les catégories de pensée, leurs enchaînements logiques ou pas, sont autant d'occasions d'oublier ce que chacune de celles-ci en sa généralité ne peut que perdre de vue, l'existence particulière en son instant et son lieu, en sa finitude - en son infini, aussi bien. Mais le moindre morceau de pain, la moindre nuée dans le ciel, n'en demeurent pas moins eucharistiques, un fait que les peintres savent parfois signifier. Et que la poésie, plus radicale que l'art, étant affaire de mots, peut, elle, non seulement exprimer mais redécouvrir, alors que le parler conceptuel, qui l'environne et qu'elle récuse, risque d'en effacer jusqu'à la mémoire.

Comprenons d'abord que dans l'expérience religieuse, quand elle s'établit sur la base d'une croyance, le sentiment de la transcendance que cette sorte de foi offre d'éprouver, c'est aussi bien et tout aussitôt la captation de cette profondeur par des mythes. Et qu'en ceux-ci les représentations, les affirmations, les figures, entretiennent entre elles des relations partiellement explicitées, conceptualisées, ce qui affaiblit et dénature l'expérience de 
la présence, privant de leur plénitude possible bien des moments pourtant essentiels dans la vie de la personne croyante. L'amour, par exemple, le simple amour naturel, se heurta à travers les siècles, dans son élan vers autrui, aux bizarres interdits de la morale que les religions mettaient en place, à cause de leur fatale idéologie. Il était alors difficile de comprendre que ceux ou celles que l'on aimait étaient plus et tout autres que les façons d'être et de vivre que l'on avait consigne d'attendre de leurs actes, de leurs désirs. Découverte que firent, cependant, et rapportèrent par bribes les romanciers, dès Cervantès, dès l'Arioste, sous le couvert encore des orthodoxies de leurs temps. À l'écoute de l'authentique infini là où il s'obstine, dans la fiction, ils comprenaient qu'il importe de dégager l'appréhension des personnes et même des choses de tout a priori décidé par une pensée analytique, même si celle-ci a fait appel aux prestiges de religions qui disent parler de la transcendance.

Il importe de dégager la réalité, la simple réalité, du voile dont cette pensée la recouvre. Or, cette découverte de la vraie nature, toute immanente, du transcendant, ce raccordement de son infini à l'existence ordinaire, c'est ce que la poésie peut, cette fois directement, accomplir ou à tout le moins tenter, puisque dans le poème le rythme qui noue les mots, la musique des vers, le pas que prennent les assonances sur les structures logiques, transgressent dans les vocables les exigences - et donc le plan - de leurs contenus conceptuels, rouvrant ainsi, à travers certes beaucoup de pièges, la voie de rencontres enfin véridiques. D'où suit que l'intuition qu'il fallait avoir quand retomba l'enfièvrement romantique, dernière grande époque de la croyance, ce fut que le besoin que l'on éprouvait de préserver l'être de la réalité immédiate, c'était aussi l'occasion de prendre conscience de ce qu'est la poésie en son vouloir et son pouvoir essentiels, jusqu'en ces années offusqués par la pensée dominante. Villon ou Racine, ou Leopardi ou Keats, étaient poètes, grands poètes, mais ils ne pouvaient percevoir clairement le vrai lieu de leur combat, ils s'engageaient donc dans des causes plus en surface, d'où d'ailleurs les disparités que l'on constate entre leurs pensées de la poésie. Ce n'est que quand le religieux chancela qu'il devint possible de discerner le poétique en sa différence, la poésie en son être propre.

Le génie de Baudelaire aura été d'avoir eu, le premier, cette intuition du plein de la poésie mais aussi d'avoir su en explorer le possible, l'éprouvant comme non l'éclair qui peut traverser la rêverie d'un artiste mais d'emblée comme un travail à porter loin dans la nuit de l'être psychique, là où les intrications de la morale en place, conceptuelle, vouent l'existence aliénée aux apories, aux conflits sans issue, aux espoirs qui s'obstinent puis renoncent. Cette intuition, une lumière d'abord lueur, la petite flamme qui va faire bouger les ombres dans ces lieux souterrains qu'avait, remarquons-le, pressentis l'imaginaire des romans noirs. Un vœu d'illumination, de présence, mais le consentement, sous un ciel aujourd'hui «bourbeux et noir", à une recherche qui sera tout un long moment sans repères. Le travail de la poésie, transgres- 
sion du plan de la représentation, ne peut être que difficile, puisque c'est au cœur même du conceptuel, c'est-à-dire à bien des niveaux de la langue, de la culture, des savoirs, et donc au plus secret de l'existence vécue qu'il doit avoir lieu, assumant les limitations de la personne, affrontant ses fatalités, revivant ses drames, transfigurant ses désirs. Et la grandeur de l'auteur des Fleurs $d u$ mal, c'est précisément d'avoir compris qu'il fallait qu'il en soit ainsi, et, courageusement, de ne pas s'être dérobé à une tâche qui ne pouvait que le vouer, entre autres misères, à l'incompréhension de ses proches.

Baudelaire a reconnu dans les situations de son existence la plus intime - structures œdipiennes, énigmes de l'être maternel, fatalités d'un tempérament, un milieu social hostile à toute déviance - les données même de son travail de poète, le matériau de ce qu'il dira son alchimie. Il a fait de son rapport difficile, orageux, tout d'ambivalence, avec sa compagne, Jeanne Duval, le creuset de cette alchimie non plus de la matière mais de la vie, il a atteint, aussi bien, au moins en des instants dont «Le Balcon» est exemple, à une rejonction de la finitude et de l'être qui est bien ce que la poésie promet et que voici donc qu'elle donne : la preuve en est faite par ce poème. Mais ce n'est pas un lieu, cette réflexion sur son siècle, pour s'arrêter davantage à Baudelaire, c'est-à-dire à l'homme qu'il fut ou à son œuvre complexe et même contradictoire. Ce que présentement je veux retenir de lui, c'est la révolution qu'ont commencée sa pensée et tout autant si ce n'est d'abord sa pratique d'une écriture imprégnée de vie quotidienne. Un élargissement, un enfièvrement, de l'emploi des mots bien faits pour «changer la vie», comme a dit le plus intense et le plus déterminé des disciples de ce poète.

Les mots ont été depuis l'aube de l'Occident les auxiliaires de sa pensée dont la vocation est analytique, soit comme structure de connaissance, soit pour diriger l'action et d'ailleurs aussi la restreindre. Chargés chacun d'un concept, quand ce n'est pas de plusieurs, ils ont mis leurs capacités relationnelles au service des signifiances avec lesquelles cette pensée bâtit ce que nous dénommons notre monde. Et de ce fait ils sont, je l'ai déjà rappelé, c'est toute l'idée de la poésie, ce qui nous prive du savoir de la finitude et de l'harmonie que ce savoir permettrait dans le rapport à soi et aux autres. Mais comment ne pas percevoir dans les mots les plus simples de l'exister quotidien un reste de la présence immédiate de ce qui est, l'originelle, celle qu'on éprouvait dans l'enfance devant des arbres, des pierres ou dans des échanges avec des proches? C'est cette aptitude des grands vocables à l'évocation d'une pleine réalité qui nous saisit, brusquement, qui nous émeut, parfois même nous bouleverse, à l'écoute de certains vers.

Les mots ont donc un pouvoir; mais ils sont tenus sous contrôle. Ce que l'on appelle la rhétorique n'a eu pour fonction, depuis les débuts en Grèce de la pensée conceptuelle, que de les garder soumis à la tâche de coordonner, analyser, signifier : l'éloquence n'étant que la façon la plus habile autant qu'insidieuse de priver l'élan poétique de sa conscience de soi. Et combien 
l'incessant travail des rhéteurs aura-t-il été efficace! Encore à l'époque du Romantisme, quand beaucoup de poètes savent pourtant percevoir le souffle de l'unité dans les vies, Victor Hugo imagine qu'il suffit d'employer des mots plus nombreux pour que soit revigoré le rapport au monde. Il veut différencier la représentation, et c'est parce qu'il oublie, au moins à des moments, que c'est la présence qui compte.

Mettre "un bonnet rouge au vieux dictionnaire», ajouter encore aux aspects par lesquels les concepts abordent les choses, ce n'est certes pas ce que Baudelaire a voulu, il a bien vu que c'est par leur mémoire du référent et non par le jeu de leurs signifiés que les mots peuvent rénover les existences; et que c'est parce qu'elle leur restitue ce pouvoir que la forme est, en poésie, créatrice : les délivrant de l'obligation de ne faire que signifier. Le mot, le grand mot libre et résonnant du "Balcon», du "Cygne», est donc, en somme, le cœur de la poésie, son grand possible apport à cette nouvelle parole qu'ont voulue Baudelaire, Rimbaud, déjà Leopardi ou Wordsworth, déjà Nerval, bientôt Yeats en son autre langue : il est le lieu du combat qu'il importe de continuer. Si le XIX siècle a un avenir à ce plan du "changer la vie" qui me paraît l'essentiel, c'est parce qu'on aura su reconnaître le mot, redevenant poésie, comme l'essentiel de son legs; et qu'il est d'emblée ce combat, d'emblée ce suprême devoir de l'être parlant en proie aux contradictions, voire aux apories, du langage.

Et que c'est difficile, se battre ainsi, aussi bien! Aller à l'arbre dans le mot "arbre», cela commence aisément quand le vers décasyllabe ou alexandrin prennent ce nom ou tout autre dans leur majesté augurale, mais c'est aussi quitter ce refuge, le dictionnaire, entrer là où des êtres vivent, et vont mourir, il va donc falloir affronter l'ordalie de la finitude, or, de toujours, l'esprit - ou disons plutôt l'intellect - se sont dérobés à la tâche, c'est une paresse spirituelle qui n'est pas prêt de cesser.

Le XIX ${ }^{\mathrm{e}}$ siècle de la poésie n'a pas eu beaucoup d'héritiers dans le $\mathrm{XX}^{\mathrm{e}}$. Il est même frappant de constater que, dressant de plus en plus fortement Mallarmé contre Baudelaire, reprenant à frais nouveaux l'antique dessein de la rhétorique, la littérature, cet antique déni de la poésie, a, réserve faite du surréalisme et de Proust, bien clairement compris que c'était le mot son péril : d'où une immense manœuvre dans les réseaux de laquelle l'heure présente est en risque d'étouffement. Cette manœuvre? Décider que le mot, qui paraît comme tel dans le travail de la forme sur la parole, doit être mis en rapport, non plus avec ce qu'il nomme dans l'au-dehors du langage, mais avec tous les autres mots qui sont dans la langue. Puisque la belle forme qu'on a donnée à la phrase a détourné celle-ci de sa signifiance, laquelle s'employait à une action ou une recherche et vouait donc ses mots à un certain sens, pourquoi ne pas s'attacher dans ceux-ci au surcroît sur ce sens de leurs signifiés et signifiances possibles, on pourrait ainsi s'évader du triste vécu, on prendrait abri parmi des ombres de signifiances aussi innombrables que délivrées d'avoir 
à se vérifier dans la vie? Le $\mathrm{XX}^{\mathrm{e}}$ siècle a hérité de l'intérêt qu'éveille le mot, mais c'est pour ne voir en ce nom propre des choses qu'une plaque tournante de concepts. Grand refermement sur de l'abstraction à nouveau, et que de périls, dans cet espace de rien que de la fiction! On ne saura plus se défendre des idéologies, la grande parole, celle qui fait de la vie un lieu d'alliance entre d'abord des présences, sera victime, encore une fois.

Il faut prêter attention à ce qui s'est joué dans Les Fleurs du mal entre «Spleen et Idéal» et les «Tableaux parisiens» : entre «Correspondances» et «Le Cygne». Il en va de la poésie. De son apport qui ne pourrait être que décisif au ressaisissement qu'il nous faut tenter.

Yves Bonnefoy est poète et essayiste, professeur honoraire au Collège de France; son ouvrage Sous le signe de Baudelaire (Gallimard, 2011) réunit quinze essais consacrés au poète et qui s'échelonnent sur près de cinquante années. 